\title{
ESTUDO DO COMPORTAMENTO FLUIDODINÂMICO DE GRÃOS DE PÓLEN APÍCOLA EM LEITO FLUIDIZADO
}

\author{
M. C. LINHARES ; L. D. M. SANTOS, M. M. PRADO \\ Universidade Federal de Sergipe, Departamento de Engenharia Química \\ E-mail para contato: milena.cabral@yahoo.com.br
}

\begin{abstract}
RESUMO - O pólen apícola é rico em compostos fenólicos e antioxidantes que o tornam um produto com alto valor agregado. A fim de viabilizar o emprego da secagem em leito fluidizado para o beneficiamento dos grãos de pólen, o presente trabalho visa à caracterização física e o estudo do comportamento fluidodinâmico destes grãos em leito fluidizado. Ensaios fluidodinâmicos foram realizados com diferentes cargas do leito (400 a $1000 \mathrm{~g}$ ), com diferentes diâmetros de partícula $\left(d_{p}=\right.$ $1,29 ; 1,55$ e $2,03 \mathrm{~mm})$ e diferentes teores de umidade $(7,12$ e $20 \%$ b.u.). Foram determinados os parâmetros fluidodinâmicos na mínima fluidização. Verificou-se que o comportamento fluidodinâmico não foi afetado nem pela carga, nem pelo teor de umidade. Entretanto, a velocidade mínima de fluidização aumenta proporcionalmente com o diâmetro da partícula, ao passo que a porosidade mínima diminui. Ademais, obtiveram-se bons índices de efetividade de fluidização em todos os casos.
\end{abstract}

\section{INTRODUÇÃO}

O pólen apícola é um produto da apicultura, rico em substâncias nutricionais valiosas, utilizado como alimento humano e associado ao bem-estar e equilíbrio funcional (VazquezQuintal et al., 2012). In natura, apresenta alto teor de umidade entre 18 e $25 \%$ b.u., o qual deve ser reduzido para $4 \%$ b.u, segundo a legislação vigente (Brasil, 2001). A secagem de grãos de pólen é essencial para a preservação da qualidade do produto, reduzindo sua atividade metabólica e a deterioração por microorganismos e assegurando a conservação de seus compostos bioativos, contribuindo assim para a obtenção de um produto com alto valor agregado.

Entretanto, o principal problema detectado no processo de produção de pólen apícola no Brasil está relacionado à etapa de secagem. Estudos (Correia-Oliveira, 2008; Ribeiro e Silva, 2007) mostram que o produto processado comumente não atende às especificações de qualidade do produto, com respeito ao teor de umidade. Isto se deve ao uso, ainda hoje, de técnicas rudimentares, como a secagem natural ou em estufas. Há, portanto, a necessidade da adoção de técnicas de secagem mais rápidas e eficientes que reduzam o consumo de energia e os custos do processo e assegurem a preservação da qualidade do produto, contribuindo também para uma maior produtividade.

O secador de leito fluidizado aparece como uma potencial alternativa de equipamento compacto e eficiente para a secagem de grãos de pólen. Existem diversas vantagens tecnológicas e econômicas no uso do leito fluidizado, podendo ser citadas: o aumento da área de transferência de calor e massa e, consequentemente, da taxa de secagem, o aumento da capacidade de processamento, a homogeneidade dos produtos secos em termos do teor de umidade e 
temperatura, a eficiência e a facilidade de instrumentação e controle do secador.

O emprego desta técnica requer a compreensão dos aspectos fluidodinâmicos do secador e a influência das características físicas do pólen na fluidização (tamanho de partícula, umidade, carga inicial). Estas informações baseiam o projeto do secador, permitindo a predição da capacidade do soprador, da produtividade e condições de estabilidade do leito. Logo, este trabalho tem como objetivos a caracterização física dos grãos de pólen e o estudo do comportamento fluidodinâmico do leito formado por este material, a fim de viabilizar o emprego da secagem do pólen apícola em leito fluidizado.

\section{METODOLOGIA}

\subsection{Material}

Foram utilizados grãos de pólen apícola provenientes de apiários localizados na Associação dos Concessionários do Distrito de Irrigação do Platô de Neópolis no estado de Sergipe. A origem botânica das amostras é, em sua maioria, de coqueiro (Cocos nucifera L.), e a umidade inicial do pólen in natura foi de aproximadamente 0,21 b.u..

\subsection{Equipamento}

O estudo do comportamento fluidodinâmico dos grãos de pólen foi realizado numa típica unidade experimental de leito fluidizado, conforme mostrado na Figura 1.

O fluxo de ar é fornecido através de uma tubulação de 2 in por um compressor (EBERLE, modelo B 100, de 4 HP de potência). A medida de vazão do ar é realizada através da leitura da diferença de pressão em um manômetro em U com água como fluido manométrico, acoplado a um medidor de vazão do tipo placa de orifício previamente calibrado. $\mathrm{O}$ ar de secagem pode ser aquecido num aquecedor elétrico de $4500 \mathrm{~W}$, passando por uma câmara plenum e atingindo o leito de partículas. Ao longo do leito existem duas tomadas de pressão, conectadas a um manômetro em U, a partir do qual pode-se monitorar a queda de pressão. Ademais, há um coletor de amostra na base do leito. O leito consiste de uma coluna com $11 \mathrm{~cm}$ de diâmetro interno e $50 \mathrm{~cm}$ de altura, construído em acrílico transparente, para permitir a visualização do regime fluidodinâmico.

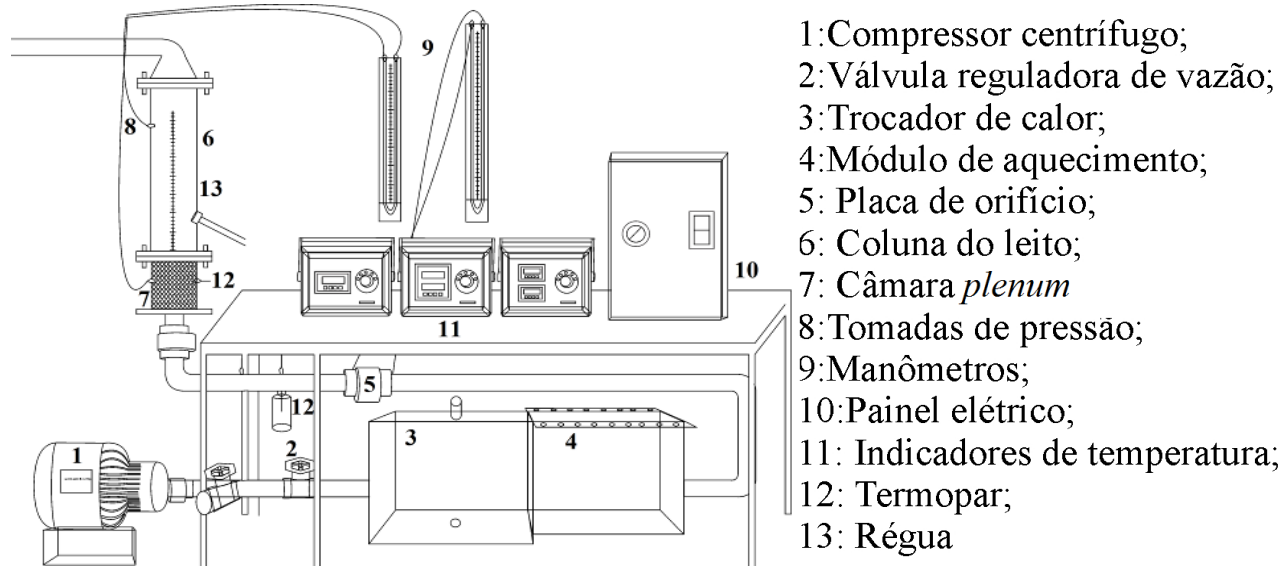

Figura 1 - Ilustração do aparato experimental. 


\subsection{Metodologia experimental}

Caracterização física: A análise granulométrica foi realizada através de peneiramento, utilizando peneiras da BERTEL montadas de forma a ter uma redução gradativa das aberturas $(2,36,1,70,1,40,1,18$, e $0,05 \mathrm{~mm})$. Para cada fração granulométrica foram medidas as dimensões dos grãos de pólen, em termos dos diâmetros mínimo, máximo e médio das partículas, através da análise de imagem com o uso do software Image-Pro Plus ${ }^{\circledR}$ versão 6.0. A esfericidade $(\phi)$ foi então determinada pela razão entre os diâmetros médio e máximo das partículas. A partir da massa das amostras e os correspondentes valores de volume, calculados com base nas dimensões obtidas via análise de imagens, a massa específica das partículas $\left(\rho_{\mathrm{p}}\right)$ foi também determinada.

Análise fluidodinâmica do leito: Primeiramente, o leito foi empacotado com uma dada carga de partículas. Então, a altura inicial do leito $\left(L_{0}\right)$ foi medida numa escala graduada fixada na coluna. Em seguida, o soprador foi ligado, anotaram-se os valores de temperaturas registrados pelos termopares localizados após a placa de orifício e na câmara plenum. Ao longo do experimento foram medidas as quedas de pressão na placa de orifício e no leito, bem como a altura do leito e as temperaturas registradas pelos termopares em função da vazão de ar. Mediramse também a temperatura e umidade relativa ambientes, no início e no final do processo, a fim de avaliar as propriedades físicas do ar. Todos os experimentos foram realizados em triplicata com diferentes frações granulométricas $(2,36-1,70 ; 1,70-1,40 ; 1,40-1,18 \mathrm{~mm})$, cargas iniciais (400 a $1000 \mathrm{~g})$ e teores de umidade $(7,12,17,3 \%)$. A velocidade mínima de fluidização $\left(v_{m f}\right)$ foi determinada graficamente com os dados da queda de pressão em função da velocidade superficial decrescente do ar. A porosidade mínima de fluidização foi calculada através da Equação (3). Calcularam-se ainda os parâmetros de qualidade de fluidização: o número de Froude $(F r)$ e o Índice de efetividade da fluidização (IF) utilizando as Equações (4) e (5), respectivamente.

$$
\begin{aligned}
& \varepsilon_{m f}=1-\frac{m_{a}}{L_{m f} A\left(\rho_{p}-\rho_{f}\right)} \\
& F r=\frac{v_{m f}^{2}}{g d_{p}} \\
& I F=\frac{-\Delta P_{m f}}{\left(g m_{p} / A\right)}
\end{aligned}
$$

onde: $A$ - área da seção reta do leito $\left(\mathrm{m}^{2}\right) ; L_{m f}$ - altura na mínima de fluidização $(\mathrm{m}) ; \rho_{\mathrm{f}}$ - massa específica do fluido $\left(\mathrm{kg} / \mathrm{m}^{3}\right) ; g$ - aceleração da gravidade $\left(\mathrm{m}^{2} / \mathrm{s}\right) ; d_{p}$ - diâmetro médio da partícula $(\mathrm{m}) ;\left(-\Delta P_{m f}\right)$ - queda de pressão na mínima de fluidização $(\mathrm{Pa})$.

\section{RESULTADOS E DISCUSSÃO}

\subsection{Caracterização física}

Os resultados típicos da distribuição granulométrica do pólen apícola in natura obtidos via análise de peneiras são apresentados na Tabela 1, bem como os valores de diâmetro projetado $\left(d_{\text {proj }}\right)$, obtidos pelo programa Image-Pro Plus ${ }^{\circledR}$, a esfericidade e a massa específica calculadas. 
Tabela 1 - Resultados de caracterização física das partículas

\begin{tabular}{|c|c|c|c|c|c|c|c|c|c|c|}
\hline $\begin{array}{c}\text { Peneira } \\
(\mathbf{m e s h})\end{array}$ & $\begin{array}{c}\boldsymbol{d +} \\
(\mathbf{m m})\end{array}$ & $\begin{array}{c}\boldsymbol{d}- \\
(\mathbf{m m})\end{array}$ & $\begin{array}{c}\boldsymbol{d}_{\boldsymbol{p}} \\
(\mathbf{m m})\end{array}$ & $\boldsymbol{x}(\boldsymbol{\%})$ & $\begin{array}{c}\boldsymbol{X}\left(\boldsymbol{d}_{\boldsymbol{p}}\right) \\
(\boldsymbol{\%})\end{array}$ & $\begin{array}{c}\mathbf{1 - X}\left(\boldsymbol{d}_{\boldsymbol{p}}\right) \\
(\boldsymbol{\%})\end{array}$ & $\begin{array}{c}\boldsymbol{d}_{\text {proj }} \\
(\mathbf{m m})\end{array}$ & $\boldsymbol{\Phi}$ & $\begin{array}{c}\boldsymbol{\rho}_{\boldsymbol{p}} \\
\left(\mathbf{g} / \mathbf{c m}^{3}\right)\end{array}$ & $\begin{array}{c}\text { Grupo } \\
\text { Geldart }\end{array}$ \\
\hline$-6+8$ & 3,32 & 2,36 & 2,84 & 16,61 & 16,61 & 83,39 & 3,00 & 0,88 & 0,64 & $\mathrm{D}$ \\
\hline$-8+10$ & 2,36 & 1,70 & 2,03 & 55,70 & 72,31 & 27,69 & 2,22 & 0,87 & 0,78 & $\mathrm{D}$ \\
\hline$-10+12$ & 1,70 & 1,40 & 1,55 & 15,64 & 87,96 & 12,04 & 1,78 & 0,86 & 0,97 & $\mathrm{D}$ \\
\hline$-12+14$ & 1,40 & 1,18 & 1,29 & 5,79 & 93,75 & 6,25 & 1,41 & 0,85 & 1,11 & $\mathrm{D}$ \\
\hline$-14+16$ & 1,18 & 1,00 & 1,09 & 1,68 & 95,43 & 4,57 & 1,11 & 0,86 & 1,39 & $\mathrm{D}$ \\
\hline$-16+32$ & 1,00 & 0,50 & 0,75 & 3,74 & 99,17 & 0,83 & 0,77 & 0,84 & 1,54 & $\mathrm{~B}$ \\
\hline Fundo & 0,50 & - & 0,25 & 0,83 & 100,00 & 0,00 & - & - & - & - \\
\hline
\end{tabular}

A análise granulométrica indica que $55,7 \%$ da massa total da amostra ficaram retidas na peneira de 10 mesh, enquanto $27,7 \%$ atravessaram essa peneira, sendo essa fração da massa dos grãos pólen formada por partículas menores que $1,70 \mathrm{~mm}$. Somente $0,8 \%$ da massa total da amostra atravessam a peneira de 32 mesh e depositam-se no fundo do equipamento. O diâmetro médio de Sauter $\left(d_{S}\right)$ é comumente utilizado em associação a fenômenos interfaciais por mensurar a razão volume/superfície das partículas de uma amostra. Para a amostra em estudo, o diâmetro de Sauter foi determinado: $d_{S}=1,73 \mathrm{~mm}$.

Verifica-se a predominância de partículas com diâmetro médio de peneira de 2,03 mm. Como a diferença entre a fração predominante e as demais frações é acentuada, o estudo da influência da carga sobre o comportamento fluidodinâmico foi realizado com esta fração.

Os valores de esfericidade obtidos foram razoavelmente elevados. Dessa forma, verificou-se que o método adotado para o cálculo da massa específica a partir do diâmetro da esfera equivalente, obtido através da análise de imagens, é adequado, pois as irregularidades da forma da partícula não ocasionaram grandes erros.

A massa específica aumenta com a diminuição do tamanho das partículas. Tal resultado indica que as menores partículas possuem menor volume de vazios, de modo que a massa diminui com a redução de tamanho do material em uma ordem de magnitude menor que a redução do volume de cada partícula.

Utilizaram-se os critérios propostos por Geldart (1973) para classificar as partículas constituintes da amostra. Verificou-se que a maior parte do material é composta por partículas da classe D, que se caracterizam pelo grande tamanho ou elevada densidade, apresentando a fluidização do tipo agregativa com surgimento de "sluggings", produzindo jatos em vez de bolhas durante a fluidização. Apenas as partículas retidas na peneira de 32 mesh foram classificadas como pertencentes ao grupo B que conduzem a uma expansão curta e borbulhante quando fluidizadas.

\subsection{Comportamento fluidodinâmico}

Na Figura 2 são apresentados os resultados típicos de queda de pressão no leito em função da velocidade crescente e decrescente do ar obtidos para os grãos de pólen de diâmetro médio de $2,03 \mathrm{~mm}$, nos ensaios realizados com a carga de 1,0 kg. A análise da influência da carga de partículas $\left(m_{0}\right)$ pode ser feita a partir das curvas de defluidização apresentadas na Figura 3. 


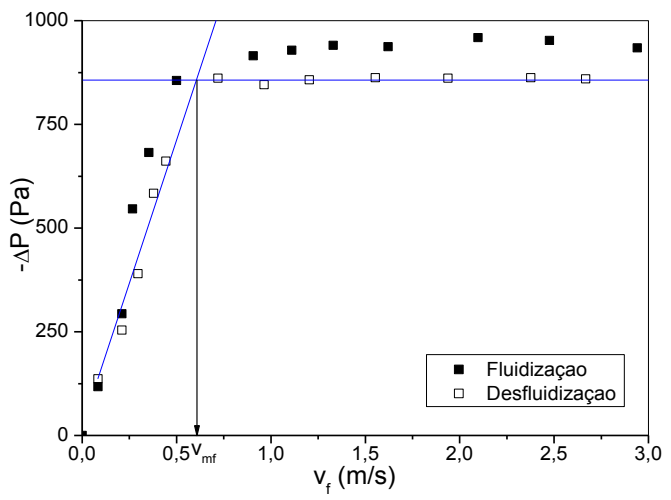

Figura 2 - Queda de pressão no leito em função da velocidade superficial do ar para a carga inicial de $1000 \mathrm{~g} \operatorname{com} d_{p}=2,03 \mathrm{~mm}$.

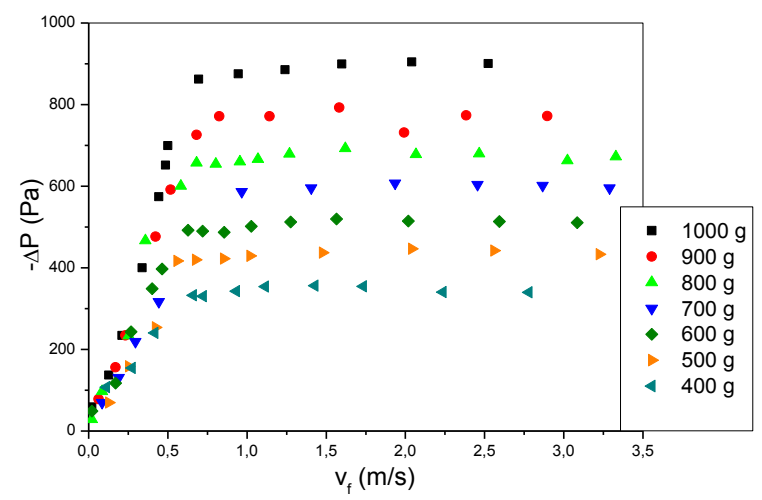

Figura 3 - Queda de pressão no leito em função da velocidade superficial do ar, para diferentes cargas de material particulado com $d_{p}=2,03 \mathrm{~mm}$ e $\mathrm{X}=8 \%$ b.u..

A partir da Figura 2 pode-se observar um comportamento típico de leito fluidizado, com a presença de uma região de leito fixo, com a queda de pressão aumentando linearmente com a velocidade do ar, até atingir o ponto de mínima fluidização, quando a força de arrasto exercida sobre o material particulado é suficiente para suspender o leito e a partir do qual a queda de pressão no leito permanece praticamente constante com o aumento da velocidade do ar, aproximadamente igual ao peso aparente do material particulado por unidade de área do leito. Nota-se ainda que o regime de fluidização foi atingido sem passar por um pico de queda de pressão, decorrente do efeito das forças interparticulares. Isso pode ser atribuído à técnica de empacotamento não denso adotada, de modo que não foi necessária energia adicional do fluido para superar a força coesiva entre as partículas e causar a expansão do leito. De acordo com Khoshtaghasa et al. (2007), o decréscimo repentino de queda de pressão ocorre com partículas grandes e com baixa esfericidade, o que não é o caso do pólen, conforme apresentado na Tabela 1.

Como esperado, os valores de queda de pressão a velocidades crescentes do ar foram superiores aos valores registrados com velocidades decrescentes. Isto ocorreu, pois na fluidização ainda há influência das forças interparticulares, enquanto que na desfluidização, tais forças já foram superadas. A Figura 2 ilustra também os procedimentos para a determinação da velocidade mínima de fluidização, a partir dos dados da desfluidização, de acordo com a metodologia de Richardson (1971). Para isso, traçaram-se duas retas, uma correspondente à região de leito fixo e outra correspondente à de leito fluidizado, a interseção das duas é o ponto de mínima fluidização. $\mathrm{O}$ valor da velocidade mínima de fluidização para o experimento da Figura 2 foi de $0,615 \mathrm{~m} / \mathrm{s}$.

Conforme pode ser observado na Figura 3, como esperado, a queda de pressão requerida para a fluidização aumentou com o aumento da massa de pólen alimentada ao leito, de modo que quando a carga é aumentada de $400 \mathrm{~g}$ para $1000 \mathrm{~g}$, a energia requerida é $141 \%$ superior. Isso ocorre visto que maior energia é demandada para executar o trabalho referente a uma maior massa. Tal informação é fundamental à seleção da potência do compressor, uma vez que a capacidade de processamento na fase de projeto ou a ponderação do aumento de produtividade, em relação aos custos de gasto energético requerido. 
A análise da influência do tamanho e do teor das partículas pode ser feita a partir das curvas de queda de pressão em função da velocidade superficial decrescente do ar apresentadas nas Figuras 4 e 5, respectivamente.

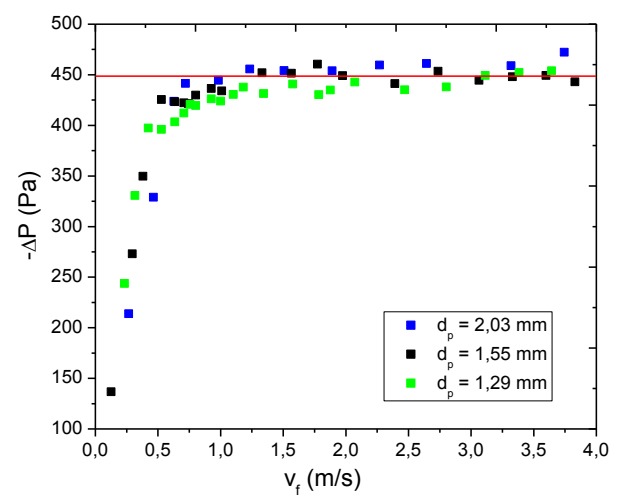

Figura 4 - Queda de pressão no leito em função da velocidade superficial do ar, para diferentes tamanhos de partícula $\left(m_{0}=500 \mathrm{~g}\right)$

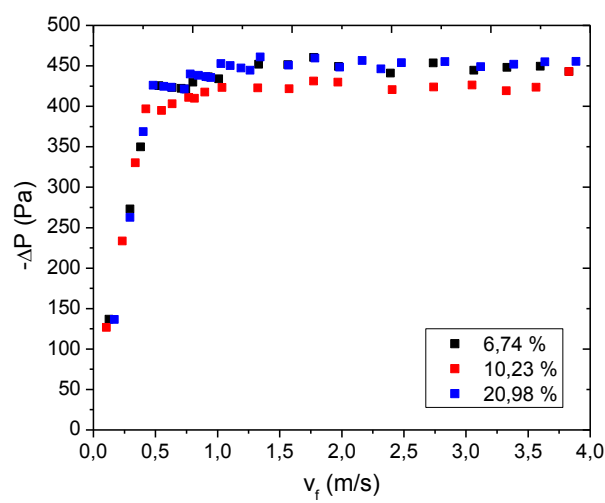

Figura 5 - Queda de pressão em função da velocidade superficial do ar para diferentes umidades iniciais $\left(d_{p}=1,55 \mathrm{~mm} ; m_{0}=500 \mathrm{~g}\right)$.

Na Figura 4, nota-se que a inclinação da reta dos pontos de leito fixo aumenta com a redução do tamanho da partícula. Já na região de leito fluidizado, os valores de queda de pressão alcançaram o mesmo patamar, cujo valor médio foi de: $-\Delta \mathrm{P}=448,82 \mathrm{~Pa}$, o qual está evidenciado pela reta no gráfico. Tal comportamento ocorre, pois na região de leito fixo há predominância das forças interparticulares, desse modo quanto menor a partícula maior o efeito das forças interparticulares, uma vez que há a redução da porosidade e o aumento do empacotamento do leito. Por sua vez, como os experimentos foram realizados com a mesma carga, a queda de pressão na fluidização é praticamente a mesma, independente do tamanho de partícula. É importante ressaltar que para a construção deste gráfico, realizaram-se experimentos com um teor de umidade médio do material de $8 \%$ b.u.

Com base nos dados apresentados na Figura 5, verifica-se que os desvios entre os dados experimentais, tanto na região de leito fixo como na de leito fluidizado, foram menores que os erros de medida, de modo que não houve influência do teor de umidade sobre o comportamento fluidodinâmico das partículas no leito, para a faixa de umidade investigada . Resultados similares foram obtidos por Wormsbecker e Pugsley (2008) para grânulos farmacêuticos

A partir do método de Richardson (1971), obtiveram-se os parâmetros de mínima fluidização e os índices de qualidade de fluidização para os experimentos realizados. Os valores encontrados para os estudos da influência da cada carga inicial, do tamanho e do teor de umidade dos grãos de pólen estão listados nas Tabelas 2, 3 e 4, respectivamente.

Verifica-se, pela Tabela 2, que não há influência da carga inicial sobre os parâmetros de mínima fluidização. A velocidade mínima de fluidização apresentou valor médio de $0,637 \pm 0,031$ $\mathrm{m} / \mathrm{s}$ e a porosidade mínima de 0,334 $\pm 0,027$. Tal comportamento condiz com os resultados obtidos por Escudero e Heindel (2011), Shaul et al. (2012) e Puspasari et al. (2013). 
Tabela 2 - Parâmetros fluidodinâmicos para diferentes cargas de partículas $\left(d_{p}=2,03 \mathrm{~mm}\right)$

\begin{tabular}{|c|c|c|c|c|c|c|c|c|}
\hline $\boldsymbol{m}_{\boldsymbol{0}}(\boldsymbol{g})$ & $\boldsymbol{W} / \boldsymbol{A}_{\boldsymbol{t}}$ & $-\boldsymbol{\Delta P}_{\boldsymbol{m f}}(\boldsymbol{P a})$ & $\boldsymbol{v}_{\boldsymbol{m} \boldsymbol{f}}(\boldsymbol{m} / \boldsymbol{s})$ & $\boldsymbol{L}_{\boldsymbol{0}}(\mathbf{c m})$ & $\boldsymbol{L}_{\boldsymbol{m f}}(\boldsymbol{c m})$ & $\boldsymbol{\varepsilon}_{\boldsymbol{m f}}$ & $\boldsymbol{F r}$ & $\boldsymbol{I F}(\boldsymbol{\%})$ \\
\hline 400 & 417 & $326 \pm 6$ & $0,653 \pm 0,043$ & 5,8 & 5,88 & $0,314 \pm 0,044$ & 21,44 & 79,2 \\
\hline 500 & 521 & $406 \pm 29$ & $0,655 \pm 0,051$ & 7,6 & 8,45 & $0,352 \pm 0,067$ & 21,57 & 78,9 \\
\hline 600 & 625 & $473 \pm 19$ & $0,621 \pm 0,085$ & 8,8 & 9,08 & $0,490 \pm 0,016$ & 19,37 & 77,1 \\
\hline 700 & 729 & $513 \pm 54$ & $0,600 \pm 0,015$ & 10,2 & 10,87 & $0,379 \pm 0,064$ & 17,73 & 72,3 \\
\hline 800 & 834 & $615 \pm 22$ & $0,617 \pm 0,069$ & 11,4 & 11,73 & $0,313 \pm 0,027$ & 19,14 & 77,1 \\
\hline 900 & 938 & $724 \pm 76$ & $0,690 \pm 0,061$ & 13,1 & 14,14 & $0,330 \pm 0,029$ & 23,90 & 79,2 \\
\hline 1000 & 1042 & $785 \pm 78$ & $0,631 \pm 0,021$ & 14,2 & 14,76 & $0,304 \pm 0,043$ & 19,97 & 77,7 \\
\hline
\end{tabular}

Tabela 3 - Parâmetros fluidodinâmicos para diferentes tamanhos de partícula, $m_{0}=500 \mathrm{~g}$

\begin{tabular}{|c|c|c|c|c|c|c|c|c|}
\hline $\begin{array}{c}\text { Peneira } \\
(\mathbf{m e s h})\end{array}$ & $\begin{array}{c}\boldsymbol{d}_{\boldsymbol{p}} \\
(\mathbf{m m})\end{array}$ & $-\boldsymbol{\Delta P}_{\boldsymbol{m} \boldsymbol{f}}(\mathbf{P a})$ & $\begin{array}{c}\boldsymbol{v}_{\boldsymbol{m} \boldsymbol{f}} \\
(\mathbf{m} / \mathbf{s})\end{array}$ & $\begin{array}{c}\boldsymbol{L}_{\boldsymbol{o}} \\
(\mathbf{c m})\end{array}$ & $\begin{array}{c}\boldsymbol{L}_{\boldsymbol{m} \boldsymbol{f}} \\
(\mathbf{c m})\end{array}$ & $\boldsymbol{\varepsilon}_{\mathbf{m f}}$ & $\boldsymbol{F r}$ & $\mathbf{I F}(\boldsymbol{\%})$ \\
\hline 10 & 2,03 & $406 \pm 29$ & $0,655 \pm 0,051$ & 7,6 & $8,45 \pm 0,35$ & $0,352 \pm 0,067$ & 21,57 & 78,9 \\
\hline 12 & 1,55 & $404 \pm 27$ & $0,506 \pm 0,036$ & 6,8 & $7,06 \pm 0,19$ & $0,400 \pm 0,034$ & 16,92 & 77,6 \\
\hline 14 & 1,29 & $404 \pm 11$ & $0,460 \pm 0,014$ & 7,2 & $7,59 \pm 0,18$ & $0,510 \pm 0,021$ & 16,70 & 77,5 \\
\hline
\end{tabular}

Tabela 4 - Parâmetros fluidodinâmicos para diferentes umidades $\left(d_{p}=1,55 \mathrm{~mm} ; m_{0}=500 \mathrm{~g}\right)$

\begin{tabular}{|c|c|c|c|c|c|c|c|}
\hline $\begin{array}{c}\text { Teor de } \\
\text { Umidade } \\
(\boldsymbol{\%} \text { b.u. })\end{array}$ & $-\boldsymbol{\Delta P}_{\boldsymbol{m} \boldsymbol{f}}(\mathbf{P a})$ & $\boldsymbol{v}_{\boldsymbol{m} \boldsymbol{f}}(\mathbf{m} / \mathbf{s})$ & $\boldsymbol{L}_{\boldsymbol{\theta}}(\mathbf{c m})$ & $\boldsymbol{L}_{\boldsymbol{m} \boldsymbol{f}}(\mathbf{c m})$ & $\boldsymbol{\varepsilon}_{\mathbf{m f}}$ & $\boldsymbol{F r}$ & $\mathbf{I F}(\boldsymbol{\%})$ \\
\hline 7 & $425 \pm 0,1$ & $0,536 \pm 0,001$ & 6,90 & $7,18 \pm 0,19$ & $0,379 \pm 0,016$ & 18,87 & 81,64 \\
\hline 12 & $383 \pm 19$ & $0,477 \pm 0,023$ & 6,70 & $6,94 \pm 0,11$ & $0,422 \pm 0,038$ & 14,96 & 73,46 \\
\hline 20 & $425 \pm 1$ & $0,527 \pm 0,022$ & 6,95 & $7,21 \pm 0,43$ & $0,381 \pm 0,038$ & 16,75 & 81,58 \\
\hline
\end{tabular}

Constata-se pelos dados da Tabela 3, que a velocidade mínima de fluidização aumentou proporcionalmente com o aumento do tamanho da partícula. Na Figura 4, pode ser observado um deslocamento dos valores de perda de carga na região de leito fixo para velocidades maiores. Isto ocorre, pois as forças inerciais associadas a partículas de maior diâmetro são maiores, sendo assim é necessário um incremento na energia cinética para provocar o deslocamento dessas partículas. Resultados similares foram obtidos por Freire et al (2008) e Gauthier et al (1999). A porosidade mínima de fluidização mostrou-se no entanto, inversamente proporcional ao diâmetro.

A partir da Tabela 4 nota-se que para a faixa estudada não há influência do teor de umidade sobre os parâmetros de mínima fluidização. A velocidade mínima de fluidização apresentou valor médio de 0,513 $\pm 0,026 \mathrm{~m} / \mathrm{s}$ e a porosidade mínima de 0,394 $\pm 0,020$. Wormsbecker e Pugsley (2008) estudaram o efeito da umidade no comportamento fluidodinâmico de partículas de placebo de 5 a $30 \%$ e verificaram um aumento, tanto da velocidade como da porosidade mínima de fluidização, com a umidade apenas para valores maiores que $20 \%$ b.u.

No estado de leito fluidizado a linha horizontal da curva de queda de pressão corresponde à grandeza peso de material particulado por unidade de área da seção transversal do leito (W/A $)_{t}$. Um dos índices que mensura a qualidade da fluidização é o índice de efetividade da fluidização (IF), que relaciona a queda de pressão e o peso por unidade de área. Calculou-se para cada condição operacional, o valor de IF e o número de Froude $(F r)$, que relaciona as energias cinética 
e gravitacional que atuam sobre as partículas. A análise dos valores do número de Froude mostra que, em todos os casos, a fluidização é agregativa $(F r>1)$, caracterizada pela formação de bolhas e "slugging", o que significa que a energia cinética das partículas supera a energia gravitacional. Esse comportamento é típico de sistemas gás-sólido, além disso, o material particulado em questão pertence à classe D de Geldart. (Kwauk et al., 2000; Singh e Roy, 2005). Em toda a faixa operacional investigada foi obtido um valor de IF em torno de $78 \%$, o que sugere que foi alcançada uma boa fluidização.

\section{CONCLUSÕES}

A queda de pressão requerida para a fluidização aumentou com a carga de pólen alimentada no leito, entretanto não houve influência da carga inicial sobre a velocidade e a porosidade de mínima fluidização. Do mesmo modo, não foi observada influência do teor de umidade das partículas sobre o comportamento fluidodinâmico do material para as condições estudadas. Mas a velocidade mínima de fluidização aumentou proporcionalmente com o tamanho da partícula

A partir do cálculo dos índices de qualidade de fluidização, verificou-se que em todas as condições ocorreu fluidização agregativa com bons índices de efetividade. Em todos os casos, o valor médio de IF obtido foi de $78 \%$.

\section{REFERÊNCIAS}

BRASIL. Ministério da Agricultura, Pecuária e Abastecimento. Instrução Normativa n ${ }^{\circ} 3$ de 19 de jan. de 2001. Diário Oficial da República Federativa do Brasil, Brasília, DF, 23 jan. 2001a. Seção 2, p. 18-23.

ESCUDERO, D.; HEINDEL, T. J.. Bed height and material density effects on fluidized bed hydrodynamics. Chem. Eng. Sci.. v. 66, ed 16, p. 3648-3655, 2011.

FREIRE, F. B.; PIRES, E. C.; FREIRE, J. T. Influência da imobilização de biomassa e do tamanho da partícula na fluidodinâmica de um reator anaeróbio de leito fluidizado. Acta Sci. Technol., v. 30, n. 1, p. 73-81, 2008.

GAUTHIER, D.; ZERGUERRAS, S.; FLAMANT, G. Influence of the particle size distribution of powders on the velocities of minimum and complete fluidization. Chem. Eng. J., v. 74, p. 181-196, 1999.

GELDART, D. Types of Gas Fluidization. Powder Technol., v.7, p. 285-292, 1973.

KHOSHTAGHAZAA, M.H. R. CHAYJAN, A.. Effect of some physical properties on fluidisation stability of grain products. Biosystems Eng., v. 98, p. 192 - 19, 2007.

KWAUK, M.; LI, J.; LIU, D.. Particulate and aggregative fluidization -50 years in retrospect. Powder Technol., v. 111, Issues 1-2, p. 3-18, 2000.

PUSPASARI, I.; TALIB, M. Z. M. W.; DAUD, R. W.; TASIRIN, S. M. .Fluidization characteristics of oil palm frond particles in agitated bed. Chem. Eng. Res. Des., v. 91, p. 497-507, 2013.

RICHARDSON, J. F. Incipient fluidization and particulate systems. Fluidization-Ed. Davidson J. F. e Harrison, D., Academic Press, Londres, 1971.

SHAUL, S.; RABINOVICH, E.; KALMAN, H..Generalized flow regime diagram of fluidized beds based on the height to bed diameter ratio. Powder Technol., v. 228, p. 264-271, 2012.

SINGH, R.K.; ROY, G.K.. Prediction of minimum bubbling velocity, fluidization index and range of particulate fluidization for gas-solid fluidization in cylindrical and non-cylindrical beds. Powder Technol., v. 159, Issue 3, p. 168-172, 2005.

VAZQUEZ-QUINTAL, P. E.; MUÑOZ-RODRIÍGUEZ, D.; MEDINA-PERALTA, S.; MOGUEL-ORDÓÑEZ, Y. B. Extraction of Organochlorine Pesticides from Bee Pollen by Matrix Solid-Phase Dispersion: Recovery Evaluation by GC-MS and Method Validation. Chromatogr., v.75, p. 923-930, 2012. 